\title{
LOGARITHMICALLY COMPLETE MONOTONICITY PROPERTIES AND CHARACTERIZATIONS OF THE GAMMA FUNCTION
}

\author{
AI-JUN LI AND CHAO-PING CHEN
}

\begin{abstract}
In this paper, the logarithmically complete monotonic properties of the functions $\prod_{i=1}^{n} \frac{\Gamma\left(x-a_{i}\right)}{\Gamma\left(x-b_{i}\right)}, \Gamma(x)^{\alpha} \Gamma\left(x-\sum_{i=1}^{n} a_{i}\right) / \prod_{i=1}^{n} \Gamma\left(x-a_{i}\right)$, and $x^{r}(e / x)^{x} \Gamma(x)$ are obtained. Some characterizations of the gamma function are deduced.
\end{abstract}

\section{Introduction}

The classical gamma function is usually defined for $\operatorname{Re} z>0$ as

$$
\Gamma(z)=\int_{0}^{\infty} t^{z-1} e^{-t} \mathrm{~d} t .
$$

It is one of the most important functions in analysis and its applications. The history and development of this function are described in detail 11 .

The psi or digamma function, the logarithmic derivative of the gamma function, and the polygamma functions can be defined $[20$, p.16] as

$$
\psi(x)=\frac{\Gamma^{\prime}(x)}{\Gamma(x)}=-\gamma+\int_{0}^{\infty} \frac{e^{-t}-e^{-x t}}{1-e^{-t}} \mathrm{~d} t
$$

or

$$
\begin{gathered}
\psi(x)=-\gamma+\sum_{n=0}^{\infty}\left(\frac{1}{1+n}-\frac{1}{x+n}\right), \\
\psi^{(k)}(x)=(-1)^{k+1} \int_{0}^{\infty} \frac{t^{k}}{1-e^{-t}} e^{-x t} \mathrm{~d} t
\end{gathered}
$$

or

$$
\psi^{(k)}(x)=(-1)^{k+1} k ! \sum_{i=0}^{\infty} \frac{1}{(x+i)^{k+1}}
$$

Received February 10, 2006; revised July 31, 2006.

2000 Mathematics Subject Classification. 33B15, 26A48.

Key words and phrases. Complete monotonicity, gamma function, logarithmically complete monotonicity.

The authors were supported in part by the Science Foundation of the Project for Fostering Innovation Talents at Universities of Henan Province, China. 
for $x>0$ and $k \in \mathbb{N}$, where $\gamma=0.57721566490153286 \ldots$ is the Euler-Mascheroni constant.

A function $f$ is said to be completely monotonic on an interval $I$ if $f$ has derivatives of all orders on $I$ which alternate successively in sign, that is

$$
(-1)^{n} f^{(n)}(x) \geq 0 \quad(x \in I ; n=0,1,2, \cdots) .
$$

If the inequality (5) is strict, then $f$ is said to be strictly completely monotonic on $I$.

"Completely monotonic functions have remarkable applications in different branches. For instance, they play a role in potential theory 8], probability theory [9, 15, 19], physics [12], numerical and asymptotic analysis [17, 28], and combinatorics [6]. A detailed collection of the most important properties of completely monotonic functions can be found in 27, Chapter IV], and in an abstract in 7]." [3, p.446]

A positive function $f$ is said be logarithmically completely monotonic on an interval $I$ if its logarithm $\ln f$ satisfies

$$
(-1)^{n}[\ln f(x)]^{(n)} \geq 0
$$

for $x \in I$ and $n \in \mathbb{N}:=1,2, \ldots$. If inequality (6) is strict, then $f$ is said to be strictly logarithmically completely monotonic. The terminology "(strictly) logarithmically completely monotonic function" was introduced in [24]. It is also shown in this paper that a (strictly) logarithmically completely monotonic function is also (strictly) completely monotonic.

In the past many articles [1, 13, 18, 25] were published providing some different properties for the ratio $\Gamma(x+1) / \Gamma(x+s)$, where $x>0$ and $s \in(0,1)$. In 1986, J. Bustoz and M.E.H. Ismail [10] established the function

$$
p(x ; a, b)=\frac{\Gamma(x) \Gamma(x+a+b)}{\Gamma(x+a) \Gamma(x+b)} \quad(a, b>0),
$$

which can be represented in terms of Gauss' hypergeometric series

$$
{ }_{2} F_{1}(a, b, c ; z)=\sum_{n=0}^{\infty} \frac{(a)_{n}(b)_{n}}{(c)_{n}} \frac{z^{n}}{n !}
$$

where $(a)_{n}=\Gamma(a+n) / \Gamma(a)$, namely,

$$
\frac{\Gamma(x) \Gamma(x+a+b)}{\Gamma(x+a) \Gamma(x+b)}={ }_{2} F_{1}(-a,-b, x ; 1), \quad(x>-a-b) .
$$

They showed that the function $p(x ; a, b)$ is completely monotonic on $(0, \infty)$. This generalized a proposition of K. B. Stolarsky [26], who obtained that $p$ is decreasing in $x$.

In 1997, H. Alzer [2] proved that the function

$$
\phi(x)=\prod_{i=1}^{n} \frac{\Gamma\left(x+a_{i}\right)}{\Gamma\left(x+b_{i}\right)}
$$


is completely monotonic on $(0, \infty)$ with $0 \leq a_{1} \leq \cdots \leq a_{n}, 0 \leq b_{1} \leq \cdots \leq b_{n}$, and $\sum_{i=1}^{k} a_{i} \leq \sum_{i=1}^{k} b_{i}$ for $k=1, \ldots, n$. This extended Bustoz's result.

Let us extend $a_{i}, b_{i}$ to all real numbers. For $a_{i}$ and $b_{i}(i=1, \ldots, n)$ are positive real numbers, define

$$
\Phi(x)=\prod_{i=1}^{n} \frac{\Gamma\left(x-a_{i}\right)}{\Gamma\left(x-b_{i}\right)} .
$$

Then we obtain that $\Phi(x)$ is logarithmically completely monotonic in the following theorems.

Theorem 1. Let $a_{i}$ and $b_{i}$ be positive real numbers, $m=\max \left\{a_{i}, b_{i}\right\}(i=1, \ldots, n)$ and $a_{i} \geq b_{i}$. Then the function $\Phi(x)$ is logarithmically completely monotonic on $(m, \infty)$.

Theorem 2. Let $a_{i}$ and $b_{i}$ be real numbers such that $0 \leq a_{1} \leq \cdots \leq a_{n}, 0 \leq b_{1} \leq$ $\cdots \leq b_{n}, m=\max \left\{a_{i}, b_{i}\right\}(i=1, \ldots, n)$ and $\sum_{i=1}^{k} a_{i} \geq \sum_{i=1}^{k} b_{i}$ for $k=1, \ldots, n$. Then the function $\Phi(x)$ is logarithmically completely monotonic on $(m, \infty)$.

Corollary 1. The function

$$
P(x ; a, b)={ }_{2} F_{1}(a, b, x ; 1)=\frac{\Gamma(x) \Gamma(x-a-b)}{\Gamma(x-a) \Gamma(x-b)} \quad(a, b>0)
$$

is strictly logarithmically completely monotonic on $(a+b, \infty)$.

In 1995, L. Maligranda et al. 21] concluded that the function

$$
x \rightarrow \Gamma(x)^{n-1} \Gamma\left(x+\sum_{i=1}^{n} a_{i}\right) / \prod_{i=1}^{n} \Gamma\left(x+a_{i}\right)
$$

$\left(a_{i}>0 ; i=1, \ldots, n\right)$ is decreasing on $(0, \infty)$. Subsequently, Alzer extended this result in [2, p.385], and obtained the necessary and sufficient condition of the statement that the function is strictly completely monotonic. The following theorem provides a slight extension of Alzer' result.

Theorem 3. Let $\alpha$ be a real number, $a_{i}(i=1, \ldots, n ; n \geq 2)$ be positive real numbers and $m=\sum_{i=1}^{n} a_{i}$. The function

$$
x \rightarrow \Gamma(x)^{\alpha} \Gamma\left(x-\sum_{i=1}^{n} a_{i}\right) / \prod_{i=1}^{n} \Gamma\left(x-a_{i}\right)
$$

is strictly logarithmically completely monotonic on $(m, \infty)$ if and only if $\alpha=n-1$.

In 2], Alzer proved that the function $F_{r}(x)=x^{r}(e / x)^{x} \Gamma(x)$ is decreasing on $(0, \infty)$ if and only if $r \leq 1 / 2$. Moreover, Alzer obtained that the function $g(x)\left(f_{1}(x)-c\right)$ is strictly completely monotonic on $(0, \infty)$ if and only if $c \leq 1 / 2$, where $g(x)$ is a strictly completely monotonic function and $f_{1}(x)=x(\ln (x)-\psi(x))$. This extended a result of E. Muldoon 
23], who proved the complete monotonicity for the special case $g(x)=1 / x$. In 2006, Alzer and Berg [4] established the completely monotonicity of function $\left[x^{a}(e / x)^{x} \Gamma(x)\right]^{b}$ for $a, b \in \mathbb{R}$ and $b \neq 0$.

Motivated by the results above, we establish several functions involving gamma and polygamma functions, and investigate their logarithmically completely monotonic properties in the following theorems.

Lemma 1.[2, p.374]] Let $\alpha$ be a real number. The function

$$
f_{\alpha}(x)=x^{\alpha}(\ln (x)-\psi(x))
$$

is strictly completely monotonic on $(0, \infty)$ if and only if $\alpha \leq 1$.

Theorem 4. Let $r$ be real number. The function

$$
F_{r}(x)=x^{r}(e / x)^{x} \Gamma(x)
$$

is strictly logarithmically completely monotonic on $(0, \infty)$ if and only if $r \leq \frac{1}{2}$; The function $\left(F_{r}(x)\right)^{-1}$ is strictly logarithmically completely monotonic on $(0, \infty)$ for $r \geq 1$.

Corollary 2. The function

$$
F_{r, \alpha}(x)=\left[x^{r}(e / x)^{x} \Gamma(x)\right]^{\alpha}
$$

is logarithmically completely monotonic on $(0, \infty)$ if and only if $r \leq \frac{1}{2}$ and $\alpha>0$.

Finally, we study the problem of characterizing $\Gamma(x)$ by means of the logarithmically completely monotonic functions related to $\Gamma(x)$. From Theorem 4, we obtain the results as follows.

Theorem 5. If function $x^{1 / 2}(e / x)^{x} f(x)$ is logarithmically completely monotonic on $(0, \infty)$ and that $f\left(x_{k}\right)=\Gamma\left(x_{k}\right)$ for each point $x_{k}$ in an increasing sequence $\left\{x_{k}\right\} \subset(0, \infty)$ for which $\sum\left(1 / x_{k}\right)$ diverges, then $f(x)=\Gamma(x), 0<x<\infty$.

Corollary 3. If function $(f(x))^{-1}$ is logarithmically completely monotonic on $(0, \infty)$ for $n=2,3, \ldots$ and that $f\left(x_{k}\right)=\Gamma\left(x_{k}\right)$ for each point $x_{k}$ in an increasing sequence $\left\{x_{k}\right\} \subset(0, \infty)$ for which $\sum\left(1 / x_{k}\right)$ diverges, then $f(x)=\Gamma(x), 0<x<\infty$.

Remark 1. The function $(\Gamma(x))^{-1}$ is logarithmically completely monotonic on $(0, \infty)$ for $n=2,3, \ldots$.

Moreover, the function $\Gamma(x)$ can be characterized in the following way $[$, p.14]: $f(x)=\Gamma(x) \quad(0<x<\infty)$ if and only if

1. $f(1)=1, f(x+1)=x f(x), \quad 0<x<\infty$;

2. $f(x)$ is defined and logarithmically convex for $0<x<\infty$. 
Requirement (2) can be modified by logarithmically completely monotonicity properties. The result is as follows.

Theorem 6. Suppose that

1. $f(1)=1, f(x+1)=x f(x), \quad 0<x<\infty$;

2. $f(x)$ is logarithmically completely monotonic on $(0, \infty)$.

Then $f(x)=\Gamma(x), 0<x<\infty$.

\section{Proofs of Theorems}

Proof of Theorem 1. Taking logarithm and differentiation yields

$$
\begin{aligned}
(\ln \Phi(x))^{\prime} & =\sum_{i=1}^{n} \psi\left(x-a_{i}\right)-\sum_{i=1}^{n} \psi\left(x-b_{i}\right) \\
& =\int_{0}^{\infty} \frac{\sum_{i=1}^{n} e^{-\left(x-b_{i}\right) t}-\sum_{i=1}^{n} e^{-\left(x-a_{i}\right) t}}{1-e^{-t}} \mathrm{~d} t \\
& =\int_{0}^{\infty} \frac{\sum_{i=1}^{n}\left(e^{b_{i} t}-e^{a_{i} t}\right)}{1-e^{-t}} e^{-x t} \mathrm{~d} t
\end{aligned}
$$

Applying power series expansion of $e^{x}$ to (7), we get

$$
(\ln \Phi(x))^{\prime}=\int_{0}^{\infty} \frac{\sum_{i=1}^{n}\left(\sum_{k=1}^{\infty}\left(b_{i}^{k}-a_{i}^{k}\right) \frac{t^{k}}{k !}\right)}{1-e^{-t}} e^{-x t} \mathrm{~d} t \leq 0
$$

By (3), we have

$$
\begin{aligned}
(-1)^{m}(\ln \Phi(x))^{(m)} & =(-1)^{m}\left(\sum_{i=1}^{n} \psi^{(m-1)}\left(x-a_{i}\right)-\sum_{i=1}^{n} \psi^{(m-1)}\left(x-b_{i}\right)\right) \\
& =\int_{0}^{\infty} \frac{\sum_{i=1}^{n}\left(e^{a_{i} t}-e^{b_{i} t}\right)}{1-e^{-t}} t^{m-1} e^{-x t} \mathrm{~d} t \\
& =\int_{0}^{\infty} \frac{\sum_{i=1}^{n}\left(\sum_{k=1}^{\infty}\left(a_{i}^{k}-b_{i}^{k}\right) \frac{t^{k}}{k !}\right)}{1-e^{-t}} t^{m-1} e^{-x t} \mathrm{~d} t \\
& \geq 0
\end{aligned}
$$

The proof is complete.

In order to prove Theorem 2 we need the following lemma [22, p.10].

Lemma 2. Let $a_{i}$ and $b_{i}(i=1 \ldots, n)$ be real numbers such that $a_{1} \leq \ldots \leq a_{n}$, $b_{1} \leq \cdots \leq b_{n}$, and $\sum_{i=1}^{k} b_{i} \leq \sum_{i=1}^{k} a_{i}$ for $k=1, \ldots, n$. If the function $f$ is increasing and convex on $\mathbb{R}$, then

$$
\sum_{i=1}^{n} f\left(b_{i}\right) \leq \sum_{i=1}^{n} f\left(a_{i}\right)
$$


Proof of Theorem 2. Since the function $x \rightarrow e^{x t}(t>0)$ is increasing and convex on $\mathbb{R}$, we conclude from Lemma 2 that $\sum_{i=1}^{n}\left(e^{a_{i} t}-e^{b_{i} t}\right) \geq 0$. Therefore (9) implies

$$
(-1)^{m}(\ln \Phi(x))^{(m)} \geq 0 \quad(m=1,2, \ldots)
$$

for $x>0$, and the function $\Phi(x)$ is logarithmically completely monotonic on $(0, \infty)$.

Proof of Corollary 1. With analogous proof method as Theorem 1, we get

$$
\begin{aligned}
& (-1)^{n}(\ln P(x ; a, b))^{(n)} \\
& =(-1)^{n}\left(\psi^{(n-1)}(x)+\psi^{(n-1)}(x-a-b)-\psi^{(n-1)}(x-a)-\psi^{(n-1)}(x-b)\right. \\
& =\int_{0}^{\infty} \frac{t^{n-1} e^{-x t}}{1-e^{-t}}\left(e^{(a+b) t}+1-e^{a t}-e^{b t}\right) \mathrm{d} t \\
& =\int_{0}^{\infty} \frac{t^{n-1} e^{-x t}}{1-e^{-t}}\left(\sum_{k=2}^{\infty}\left((a+b)^{k}-a^{k}-b^{k}\right) \frac{t^{k}}{k !}\right) \mathrm{d} t>0
\end{aligned}
$$

Now we provide another method to prove Corollary 1.

For $n \geq 0$, we have that

$$
\begin{aligned}
(\ln P(x ; a, b))^{(n+1)} & =\psi^{(n)}(x)+\psi^{(n)}(x-a-b)-\psi^{(n)}(x-a)-\psi^{(n)}(x-b) \\
& =a\left(\frac{\psi^{(n)}(x)-\psi^{(n)}(x-a)}{a}-\frac{\psi^{(n)}(x-b)-\psi^{(n)}(x-b-a)}{a}\right) .
\end{aligned}
$$

By (4I), $y \longmapsto \psi^{(n)}(y)$ is strictly convex for odd $n$, the ratio

$$
\frac{\psi^{(n)}(x)-\psi^{(n)}(x-a)}{a}
$$

is increasing with $y \in(a, \infty)$. For even $n$, the function $\psi^{(n)}(x)$ is concave, and the ratio (12) is decreasing. Thus, by (11) we conclude that the sign of $(\ln P(x ; a, b))^{(n+1)}$ is $(-1)^{n+1}$, for $n \geq 0$ and $x \in(a+b, \infty)$.

Proof of Theorem 3. Let

$$
p_{\alpha}(x)=\Gamma(x)^{\alpha} \Gamma\left(x-\sum_{i=1}^{n} a_{i}\right) / \prod_{i=1}^{n} \Gamma\left(x-a_{i}\right) .
$$

It is obvious that $p_{n-1}(x)$ is strictly logarithmically completely monotonic on $(m, \infty)$ from Theorem 2.

Next, we assume that $p_{\alpha}(x)$ is strictly logarithmically completely monotonic on $(m, \infty)$. Then, we get

$$
\frac{\partial}{\partial x} \ln p_{\alpha}(x)=\alpha \psi(x)+\psi(x-m)-\sum_{i=1}^{n} \psi\left(x-a_{i}\right) \leq 0 .
$$


This implies for all sufficiently large $x$ :

$$
\alpha \leq \sum_{i=1}^{n} \frac{\psi\left(x-a_{i}\right)}{\psi(x)}-\frac{\psi(x-m)}{\psi(x)}
$$

Since $p_{\alpha}$ is completely monotonic on $(m, \infty)$, we obtain

$$
\begin{aligned}
0 & \leq\left(p_{\alpha}(x)\right)^{-2}\left[p_{\alpha}(x) \frac{\partial^{2} p_{\alpha}(x)}{\partial x^{2}}-\left(\frac{\partial p_{\alpha}(x)}{\partial x}\right)^{2}\right] \\
& =\alpha \psi^{\prime}(x)+\psi^{\prime}(x-m)-\sum_{i=1}^{n} \psi^{\prime}\left(x-a_{i}\right) .
\end{aligned}
$$

Hence, we have for $x>m$ :

$$
\sum_{i=1}^{n} \frac{\psi^{\prime}\left(x-a_{i}\right)}{\psi^{\prime}(x)}-\frac{\psi^{\prime}(x-m)}{\psi^{\prime}(x)} \leq \alpha
$$

Since

$$
\lim _{x \rightarrow \infty} \psi(x-A) / \psi(x)=\lim _{x \rightarrow \infty} \psi^{\prime}(x-A) / \psi^{\prime}(x)=1 \quad(A>0),
$$

we conclude from (14) and (15) that $\alpha=n-1$.

Proof of Theorem 4. Using Binet's formula 14, p.18, (22)], we get

$$
\begin{aligned}
\left(\ln F_{r}(x)\right)^{\prime} & =\frac{r}{x}-\ln x+\psi(x) \\
& =\frac{r}{x}-\ln x+\ln x-\frac{1}{2 x}+\int_{0}^{\infty}\left(\frac{1}{2}+\frac{1}{t}-\frac{1}{1-e^{-t}}\right) e^{-x t} \mathrm{~d} t \\
& =\frac{r-1 / 2}{x}+\int_{0}^{\infty}\left(\frac{1}{2}+\frac{1}{t}-\frac{1}{1-e^{-t}}\right) e^{-x t} \mathrm{~d} t \\
& <0 .
\end{aligned}
$$

(16) follows from $r \leq \frac{1}{2}$ and the inequality

$$
\frac{1}{2}+\frac{1}{t}-\frac{1}{1-e^{-t}}=\frac{2-t-(2+t) e^{-t}}{2 t\left(1-e^{-t}\right)}<0 \quad(0<t<\infty)
$$

Indeed, let $g(t)=2-t-(2+t) e^{-t}$, we can get $g^{\prime \prime}(t)=-t e^{-t}<0$ and $\lim _{t \rightarrow 0} g^{\prime}(t)=0$. This implies that $g(t)$ is decreasing on $(0, \infty)$. Since $\lim _{t \rightarrow 0} g(t)=0$, (17) holds. 
Taking the $n$th derivative of $\ln F_{r}(x)$, we obtain

$$
\begin{aligned}
& (-1)^{n}\left(\ln F_{r}(x)\right)^{(n)} \\
& =(-1)^{n}\left[\left(\frac{r-1 / 2}{x}\right)^{(n-1)}+\left(\int_{0}^{\infty}\left(\frac{1}{2}+\frac{1}{t}-\frac{1}{1-e^{-t}}\right) e^{-x t} \mathrm{~d} t\right)^{(n-1)}\right] \\
& =-\frac{(r-1 / 2)(n-1) !}{x^{n}}-\int_{0}^{\infty}\left(\frac{1}{2}+\frac{1}{t}-\frac{1}{1-e^{-t}}\right) t^{n-1} e^{-x t} \mathrm{~d} t \\
& >0 \quad\left(r \leq \frac{1}{2}\right) .
\end{aligned}
$$

Next, it is clear from (16) that

$$
r<x(\ln x-\psi(x)) .
$$

By Lemma 1, we obtain that $f_{1}(x)=x(\ln x-\psi(x))$ is strictly decreasing on $(0, \infty)$. Moreover,

$$
\lim _{x \rightarrow \infty} f_{1}(x)=\frac{1}{2}
$$

It follows from the representations

$$
f_{1}(x)=x \ln x-x \psi(x+1)+1
$$

and

$$
f_{1}(x)=\frac{1}{2}+\frac{1}{12 x}-\frac{\theta}{120 x^{3}} \quad(0<\theta<1) ;
$$

see [16, p.824]. Therefore, we conclude $r \leq \frac{1}{2}$.

To prove the second part, a simple calculation shows that

$$
\left(\ln \left(F_{r}(x)\right)^{-1}\right)^{\prime}=\int_{0}^{\infty}\left(\frac{1}{1-e^{-t}}-\frac{1}{t}-\alpha\right) e^{-x t} \mathrm{~d} t<0
$$

and

$$
(-1)^{n}\left(\ln \left(F_{r}(x)\right)^{-1}\right)^{(n)}=-\int_{0}^{\infty}\left(\frac{1}{1-e^{-t}}-\frac{1}{t}-\alpha\right) t^{n-1} e^{-x t} \mathrm{~d} t>0
$$

for $r \geq 1$. Since we write

$$
f(t)=\frac{1}{1-e^{-t}}-\frac{1}{t}
$$

we have $f(0+)=\frac{1}{2}, f(\infty)=1$.

This completes the whole proof.

Proof of Theorem 5. It is shown by W. Feller [15, p.671] that two functions which are completely monotonic on $(0, \infty)$ must be identical if they coincide at the points of an increasing unbounded sequence $\left\{x_{k}\right\}$ where $\sum\left(1 / x_{k}\right)$ diverges.

Since the functions $x^{1 / 2}(e / x)^{x} f(x)$ and $x^{1 / 2}(e / x)^{x} \Gamma(x)$ are both (logarithmically) completely monotonic on $(0, \infty)$, we get $f(x)=\Gamma(x)$ easily. 


\section{Acknowledgements}

The authors are indebted to the anonymous referee for many valuable comments.

\section{References}

[1] H. Alzer, Some gamma function inequalities, Math. Comp. 60 (1993), 337-346.

[2] H. Alzer, On some inequalities for the gamma and psi functions, Math. Comput. 66 (1997) $373-389$.

[3] H. Alzer and C. Berg, Some classes of completely monotonic functions, Ann. Acad. Scient. Fennicae 27 (2002), 445-460.

[4] H. Alzer and C. Berg, Some classes of completely monotonic functions, II, Ramanujan Journal 11 (2006), 225-248.

[5] E. Artin, The gamma function, Athena series, Holt, Rinehart and Winston, New York, 1964.

[6] K. Ball, Completely monotonic rational functions and Hall's marriage theorem, J. Comb. Th., Ser. B, 61 (1994), 118-124.

[7] C. Berg, J. P. R. Christensen, P. RESSEL, Harmonic analysis on semigroups. Theory of positive definite and related functions, Graduate Texts in Mathematics 100, Springer, Berlin- Heidelberg-New York, 1984.

[8] C. Berg, G. Forst, Potential theory on locally compact abelian groups, Ergebnisse der Math., 87, Springer, Berlin, 1975.

[9] L. Bondesson, Generalized gamma convolutions and related classes of distributions and densities, Lecture Notes in Statistics 76, Springer, New York, 1992.

[10] J. Bustoz and M. E. H. Ismail, On gamma function inequalities, Math. Comp. 47 (1986), 659-667.

[11] P. J. Davis, Leonhard Euler's integral: A historical profile of the gamma function, Amer. Math. Monthly 66 (1959), 849-869.

[12] W. A. Day, On monotonicity of the relaxation functions of viscoelastic meterial, Proc. Cambridge Philos. Soc. 67 (1970), 503-508.

[13] T. Erber, The gamma function inequalities of Gurland and Gautschi, Skand. Aktuarietidskr. 1960 (1961), 27-28.

[14] A. Erdélyi, et al., Higher transcendental functions, vol. 1. McGraw-Hill, New York, 1953.

[15] W. Feller, An Introduction to Probability Theory and its Applications, Vol. 2, Wiley, New York, 1966.

[16] G. M. Fichtenholz, Dierential- und integralrechnung II, Dt. Verlag Wiss., Berlin, 1978.

[17] C. L. Frenzen, Error bounds for asymptotic expansions of the ratio of two gamma functions, SIAM J. Math. Anal. 18(1987), 890-896.

[18] D. Kershaw, Some extensions of W. Gautschi's inequalities for the gamma function, Math. Comp. 41 (1983), 607-611.

[19] C. H. Kimberling, A probabilistic interpretation of complete monotonicity, Aequat. Math. 10 (1974), 152-164.

[20] W. Magnus, F. Oberhettinger, and R. P. Soni, Formulas and theorems for the special functions of mathematical physics, Springer, Berlin, 1966. 
[21] L. Maligranda, J. E. Pečarić, and L. E. Persson, Stolarsky's inequality with general weights, Proc. Amer. Math. Soc. 123 (1995), 2113-2118.

[22] A. W. Marshall and I. Olkin, Inequalities: Theory of majorization and its applications, Academic Press, New York, 1979.

[23] M. E. Muldoon, Some monotonicity properties and characterizations of the gamma function, Aequationes Math. 18 (1978), 54-63.

[24] F. Qi and Ch.-P. Chen, A complete monotonicity property of the gamma function, J. Math. Anal. Appl. 296 (2004), 603-60

[25] D. V. Slavić, On inequalities for $\Gamma(x+1) / \Gamma(x+1 / 2)$, Univ. Beograd. Publ. Elektrotehn. Fak. Ser. Mat. Fiz. 498-541 (1975), 17-20.

[26] K. B. Stolarsky, From Wythoff's Nim to Chebyshev's inequality, Amer. Math. Monthly 98 (1991), 889-900.

[27] D. V. Widder, The Laplace transform, Princeton Univ. Press, Princeton, NJ, 1941.

[28] J. Wimp, Sequence transformations and their applications, Academic Press, Nex York, 1981.

Department of Mathematics, Shanghai University, Shanghai 200444, China.

E-mail: liaijun72@163.com

School of Mathematics and Informatics, Research Institute of Applied Mathematics, Henan Polytechnic University, Jiaozuo City, Henan 454010, China.

E-mail: chenchaoping@sohu.com 